\title{
Alteridad, género, sexualidad y afectos. Reflexiones a partir de una experiencia investigativa en Colombia*
}

\author{
Mara Viveros Vigoya**
}

\begin{abstract}
Plantear la pregunta sobre la pertinencia analítica y política de utilizar categorías como sexualidad, erotismo, género y amor para hablar de prácticas de grupos sociales que no se adecúan a los presupuestos sobre los cuales se fundaron dichas categorías es un reto para investigadoras $e$ investigadores latinoamericanos que nos enfrentamos a realidades sociales muy complejas. Deseo responder al estímulo intelectual y político que suscita esta pregunta a partir de mi experiencia investigativa sobre relaciones de género y sexualidad con hombres y mujeres "negros" en Colombia, habitantes de sectores populares y medios en ciudades como Bogotá y Cali o en pequeñas ciudades como Quibdó. ${ }^{1}$

Lo primero que quisiera aclarar es que nunca he considerado a estas mujeres y hombres como parte de un grupo "tradicional" y por el contrario, siempre lo he percibido muy conectado con la modernidad, así fuera en forma parcial o paradójica. Digo parcial, porque su inclusión en los parámetros de
\end{abstract}

\footnotetext{
* Recebido para publicação em 9 de setembro de 2013, aceito em 15 de outubro de 2013.

** Profesora de la Escuela de estudios de Género y del Departamento de Antropología de la Facultad de Ciencias Humanas, Universidad Nacional de Colombia.mviverosv@unal.edu.co

1 Mientras en Bogotá el porcentaje de población que se declaró como negra o afrocolombiana en el último censo (2005) no alanza el $2 \%$, Cali es, después de Salvador de Bahía la segunda ciudad latinoamericana con mayor número de población afrocolombiana y Quibdó, una ciudad de 120 mil habitantes tiene un $82 \%$ de población que se autoidentifica como tal (Fuente: censo general 2005. Información básica, DANE, Colombia).
}

cadernos pagu (41), julho-dezembro de 2013:41-52. 
reflexiones a partir de una experiencia investigativa en Colombia

la modernidad no ha sido total, pese a su largo contacto con el medio urbano, y paradójica, porque ha sido caracterizado a la vez como un grupo de ciudadanos comunes y como parte de la Alteridad localizada en los espacios marginados de la nación. Sin embargo, "no se les ha institucionalizado como Otros de igual forma que a los indígenas" (Wade, 1997:48). Los lugares sociales que han ocupado unos y otros han sido distintos desde el período colonial (en el cual los Indios, a diferencia de los Negros gozaban de protección legal) y esta ubicación diferenciada ha tenido repercusiones tanto en las nuevas repúblicas como en unos imaginarios de nación que se prolongaron en el ámbito político de los siglos XX y XXI.

Aunque se admite que las fronteras de la dominación colonial fueron siempre igualmente fronteras sexuales - que buscaban garantizar el control de los otros racializados -, las relaciones de género y sexualidad solo cobraron importancia como temas de investigación en los estudios de raza y etnicidad en América Latina en los años 80 y 90 del siglo XX. Hasta entonces, los grupos étnico-raciales habían sido considerados únicamente desde el ángulo de la historia política, económica y social. La distinción entre Indios y Negros ha implicado a su vez aproximaciones muy diferentes a sus sexualidades: mientras la mayoría de los trabajos moviliza representaciones muy sedimentadas de la sexualidad como un eje central de las identidades negras, el tema de la sexualidad en las poblaciones indígenas parecería ser menos relevante $e^{2}$. Por el contrario se ha señalado que las jerarquías de género estarían más presentes en los grupos indígenas, hasta el punto de que muchas veces quienes son percibidos como "indios" en los confines del patriarcado moderno son generalmente las mujeres, con menor contacto con lo urbano que los hombres de sus propias comunidades. Esto es lo

\footnotetext{
2 Vale la pena señalar que esta desexualización no es uniforme y que algunas mujeres de ascendencia indígena como las llamadas "cholas" en Perú, son por el contrario hipersexualizadas (Boesten, 2008).
} 
que quiere decir Marisol de la Cadena (1995) cuando afirma que "las mujeres son más indias" que los hombres, a partir de los resultados de un estudio de caso sobre relaciones de género y relaciones interétnicas realizado en Chitapampa, una pequeña comunidad campesina situada cerca de la ciudad de Cuzco.

En el caso de los grupos negros, sus mujeres han sido excluidas del significado de la feminidad - como lo señaló con agudeza desde mediados del siglo XIX, la exesclava estadounidense Sojourner Truth - constituida con base en la experiencia de las mujeres blancas de clase media, y en consecuencia han tendido a ser menos dependientes de los salarios masculinos que las mujeres de clases medias blancas. Por su parte, la masculinidad de los hombres negros ha sido constantemente devaluada, en términos de su menor cumplimiento de los mandatos de género como principales proveedores de sus hogares y sus responsabilidades paternas, y sobreestimada en términos de su rendimiento sexual (Viveros, 2002 y 2013).

Además, en un mundo en el cual los intercambios culturales se hacen cada vez más estrechos debido al proceso de globalización, es necesario considerar que el análisis de unas formas "otras", "divergentes" de afrontar la sexualidad, el afecto, el género o el placer en el mundo contemporáneo supone hacerlo en el marco de este proceso en el cual la cultura, particularmente la "occidental", tiende a mundializarse. Muchos de los códigos contemporáneos de la moralidad sexual y de las leyes referentes a la sexualidad de los países latinoamericanos hunden sus raíces en la historia y tradición europeas, transmitidas desde el periodo colonial. Es difícil sostener hoy algunas hipótesis que señalan que existe un "afuera" aislado de Occidente y encarnado en unas poblaciones marginadas, subalternas o "no occidentales", que en el caso latinoamericano serían las indígenas y afrodescendientes. Al mismo tiempo, es necesario dejar un espacio heurístico para comprender formas de hibridación, de reapropiación, de desafío o incluso de desconocimiento voluntario o involuntario de ese proceso de "occidentalización", personificadas por estos grupos 
reflexiones a partir de una experiencia investigativa en Colombia

subalternos marcados tanto por la impronta de la colonización y la esclavización como por la de la globalización.

$\mathrm{Mi}$ experiencia investigativa en distintos contextos colombianos me ha enfrentado a la necesidad de comprender de manera más compleja el hecho de que "Occidente", "lo blanco" o "la clase media", no son realidades totales. "Occidente" y las categorías asociadas a éste también han sido "impurificadas", por decirlo de alguna manera, por esas y esos Otros. Sabemos que "Occidente" y "la Alteridad" son "realidades" que se co-construyen mutuamente (Saïd, 2003) en una relación de poder, que es al mismo tiempo prescriptiva y productiva. La Alteridad produce la racialización de los cuerpos, ubicándolos en un orden racial, social y moral que es indisociablemente sexuado. En este contexto el cuerpo cobra una relevancia singular, ya no sólo como una metáfora de lo social, sino como la superficie misma sobre la cual se inscribe la microfísica del poder (Foucault, 1991).

Indudablemente, puede parecer extraño movilizar teóricamente una aproximación foucaultiana de la sexualidad y el biopoder, fuertemente anclada en Occidente, para pensar las relaciones sexuadas en contextos con un fuerte legado histórico colonial como el latinoamericano, a sabiendas de que el punto ciego de esta perspectiva ha sido la inclusión del colonialismo y la colonialidad del poder en la Historia de la sexualidad. Sin embargo, rechazar totalmente todo concepto y marco teórico occidental porque no tuvo en cuenta la diferencia colonial puede ser una postura tan acrítica como su adopción total, sin criterios propios para definir qué es oportuno utilizar en un análisis de las sociedades locales y qué no lo es. Por ejemplo, lecturas feministas como las que propone Ann Laura Stoler en Race and the Education of Desire. Foucault's History of Sexuality and the Colonial Order of Things, señalan con pertinencia que la noción de biopoder puede ser útil no solo para referirse al sexo sino a la raza, como lo expresa Foucault en su obra publicada posteriormente a la Historia de la Sexualidad, titulada Defender la sociedad. 
En este trabajo Foucault plantea que la racionalidad política del racismo no es "hacer vivir y dejar morir", como se supone que hacen las formas de gobierno europeas a partir del siglo 18, sino ocuparse de "la función de muerte en la economía del biopoder" (Foucault, 2001:233). De esta manera, muestra que el racismo vuelve compatible la condena a muerte de personas y sectores poblacionales con el ejercicio del biopoder. Sin embargo, al señalar que "las colonias fueron uno de los laboratorios donde se probó el racismo en tanto dispositivo biopolítico de guerra" (Castro-Gómez, 2007:158), pero no el único, se ignora el hecho de que este racismo no generó culpabilidad en las sociedades occidentales sino cuando se aplicó sobre poblaciones europeas como sucedió con el nazismo a mediados del siglo XX (Césaire, Aimé, 2006).

Stoler (2005) subraya que la dominación colonial no es únicamente un asunto de racismo sino también de género y sexualidad y que las desigualdades entre los sexos han sido esenciales en la estructura del racismo colonial y la autoridad imperial. Esta importancia determina que las sanciones y prohibiciones sexuales no solo sean "útiles para delimitar las posiciones de poder sino también para prescribir las fronteras personales y públicas de la raza (Stoler, 2005:78).

La intimidad y la sexualidad continuaron siendo centrales para el orden social de las colectividades latinoamericanas en el período postcolonial, pero en un marco nuevo: el de la "nación" moderna y respetable (Wade, 2009). En efecto, el código del honor siguió operando para reproducir las desigualdades raciales y de género a pesar de que el mestizaje fue posicionado como el rasgo definitorio de las identidades nacionales latinoamericanas. Incluso en el contexto del multiculturalismo, y de su celebración de la diversidad, en vigor desde los años noventa del siglo $\mathrm{XX}$, sigue siendo significativo quién es objeto de elección de una relación sexual y quién objeto de una alianza matrimonial; qué tipo de relaciones heterosexuales $\mathrm{u}$ homosexuales se estimulan $\mathrm{o}$ se prohíben en una economía de valores y estatus eróticos, afectivos 
reflexiones a partir de una experiencia investigativa en Colombia

y económicos que tiene dimensiones tanto nacionales como transnacionales (Wade, 2009 y Viveros, 2012 y 2013).

La complejidad de las relaciones erótico-afectivas a las que hacemos alusión, exige adoptar marcos de comprensión flexibles e inductivos que nos permitan captar "otras" posibilidades de vivir lo que llamamos "sexualidad", "intimidad" o "afectividad", sin tener que remitirlas a lo "exótico", al "más allá de occidente" o "al otro lado". Esos agenciamientos, esas invenciones relacionales también se pueden dar o presentar en las capas medias, mestizas, letradas, más o menos modernizadas pero igualmente atravesadas por contradicciones, ligadas a la condición mestiza que las constituye pero que constantemente se acalla.

No obstante, vale la pena preguntarse si la sexualidad es un término con un significado universal y si tiene la misma importancia en todos los contextos sociales y geopolíticos. Todo indicaría que no y que por el contrario, evoca distintas nociones de placer, creencias personales, valores, emociones y debates diferentes sobre las opresiones y violencias a las que da lugar. La sexualidad es además, uno de los tópicos humanos que se pueden abordar desde las más diversas perspectivas, históricas, sociológicas, antropológicas, psicológicas, jurídicas, feministas, pero también desde la poesía, la ficción, las historias de vida, la música, el arte y la salud pública (Tamale, 2011). Ahora bien, ¿existen unas sexualidades latinoamericanas, colombianas, o "negras"? ¿Quién determina qué se puede calificar como tal?

Mi trabajo investigativo en distintos contextos colombianos remite a una realidad heterogénea donde "la masculinidad" y la "feminidad", "la clase media", "lo negro", "lo blanco", la "heterosexualidad" y la "homosexualidad" no son esencias, ni categorías naturales o pre-sociales, despojadas de historia, ni posiciones de sujeto o experiencias sociales uniformes $y$ monolíticas. El estudio sobre masculinidades y relaciones de género en Armenia y Quibdó (2002), me permitió entender que esas identidades y relaciones no solo son inestables desde el punto de vista de género sino que esta inestabilidad debe ser entendida 
en una escala multidimensional que articule distintas categorías de poder como la raza, la clase y la sexualidad y de cuenta de las jerarquías que se crean entre ellas. Esta experiencia me llevó a "corregir" y "revisar" las concepciones canónicas sobre las identidades de género $y$ las definiciones de masculinidad $y$ feminidad, a la luz de las evidencias contextuales del terreno y a adoptar lo que hoy llamamos una perspectiva interseccional para informar de las relaciones imbricadas de poder que construyen de estas masculinidades. Igualmente, tuve que afinar el uso de esta perspectiva, yendo más allá de las concepciones aditivas que suponen que cada una de estas jerarquías existe de modo completo, intersectándose luego con las otras "a manera de vectores", para asumir una noción más consubstancial de la interseccionalidad en la cual estos órdenes son recíprocamente constituidos.

En mi reciente investigación sobre trayectorias de ascenso social de mujeres y hombres negros residentes en Bogotá (Viveros y Gil, 2010), los procesos analizados corresponden a dinámicas de enclasamiento en las cuales se refuerzan y al mismo tiempo se trasgreden o se desplazan normas y valores de género (Butler, 2005). Las clases medias colombianas que hemos analizado incluyen el acceso restringido de algunos "Otros" y "Otras" racializados que redefinen parcialmente las fronteras de estas clases, así en su gran mayoría ellas y ellos deban reiterar y citar constantemente los mandatos axiológicos del ethos burgués, moderno, blanco y heterosexual para poder ser aceptados como parte de estas clases. Por otra parte, en el trabajo sobre heterosexualidades, anticoncepción y aborto (Viveros et al, 2011), encontramos que asumir la maternidad a edad temprana no significa necesariamente una mayor adhesión a las normas de género dominantes que definen la feminidad por su capacidad procreativa. En algunas oportunidades pueden expresar, por el contrario, una resistencia a las normas de clase que definen cuales son los calendarios adecuados para los eventos reproductivos en el curso de una vida "moderna" y "respetable". La investigación sobre clases medias negras también ha identificado algunas 
reflexiones a partir de una experiencia investigativa en Colombia

trayectorias biográficas que van a contrapelo del sentido común que supone que ascender socialmente significa necesariamente blanquearse; a la inversa, algunos de estos itinerarios muestran que se puede "devenir negro" en el proceso mismo del ascenso social, mediante el acceso a la educación superior y sin pretender instalarse socialmente como parte un nuevo grupo de poder.

Estas revisiones de las teorías a la luz del terreno local no eliminan la necesidad de vincular estos análisis, por una parte a las dinámicas transnacionales que inciden en los procesos locales, y por otra parte, a las realidades estructurales comunes a estos distintos contextos, relacionadas con la llamada "feminización de la pobreza", la racializada y jerarquizada distribución geográfica de las poblaciones en Colombia, o los modelos de desarrollo neo (liberal) y sus políticas de gobierno de la sexualidad juvenil (Viveros, 2004). Tampoco se puede ignorar el papel que desempeñan las entidades internacionales que financian las investigaciones en sexualidad, en la definición de los derroteros teóricos o metodológicos de estos estudios.

Por último, es muy importante prestar atención al modo en que se entienden los vínculos existentes entre los distintos niveles de análisis y su generalización, micro, meso y macro social. Mi experiencia investigativa me ha llevado a adoptar una perspectiva heterárquica y no jerárquica de las relaciones de poder para abordar ciertas prácticas sociales que constituyen una suerte de "zonas grises" - que escapan parcialmente a los ordenamientos de género, raza, clase y sexualidad. Documentar la existencia de los micro-agenciamientos individuales - que buscan revertir las tendencias de larga duración que constituyen las normas de género, raza y clase - no significa desconocer la lógica de los regímenes más globales y su incidencia en los más recónditos rincones de lo que llamamos 'intimidad' sino

reconocer que estos regímenes no funcionan en abstracto sino a través de tecnologías de subjetivación y regularización, como son, por ejemplo, la disciplina y la 
biopolítica, cuya operatividad debe ser investigada empíricamente en los niveles más locales (Castro Gomez, 2007:169).

Una perspectiva heterárquica de las relaciones de poder permitirá vincular en los estudios sobre sexualidad las estructuras molares de la clase, la raza y el género con las estructurales moleculares de los afectos, los sentimientos, las emociones y las representaciones estéticas, sin suponer que las segundas son lógicamente y ontológicamente dependientes de las primeras. Tomemos un ejemplo de la investigación sobre ascenso social de poblaciones negras mencionada antes, para ilustrar lo que intento plantear. Haré referencia al trabajo artístico de Liliana Angulo Cortés y Fabio Melecio Palacios, dos jóvenes artistas plásticos afrocolombianos contemporáneos cuyas propuestas socavan de distinto modo las representaciones estereotipadas de la gente negra colombiana. Mientras Liliana Angulo utiliza con gran maestría y virulencia la parodia en sus videos, exhibiciones de fotografías y performances, Fabio Melecio Palacios, disloca los significantes y significados de lo negro para crear paisajes abstractos inesperados que los relocalizan.

Liliana Angulo redobla la violencia que expresa el rechazo a rasgos fenotípicos de la población negra como el pelo crespo denominado como "cabello malo" en su proyecto "Un negro es un negro". Serie "Pelucas Porteadores". Lo hace a través de la elaboración de enormes pelucas a partir de esponjillas para lavar ollas que llevan en su cabeza las modelos negras. También ha hecho representaciones de mujeres negras cuya indumentaria es del mismo estampado que el azulejo de la cocina para explicitar el sentido común según el cual las mujeres negras y el empleo doméstico son una misma cosa. Sus representaciones de lo negro ponen en escena, con humor "negro", las tensiones $y$ ambigüedades que suscitan los estereotipos de género y raza, vinculándolos siempre con esa historia de la esclavización y la servidumbre postcolonial que muchas veces se prefiere olvidar. 
reflexiones a partir de una experiencia investigativa en Colombia

Podríamos decir que la estrategia de Angulo ha sido la que utiliza Frantz Fanon en su libro Pieles Negras, máscaras blancas cuando se llama a si mismo Negro para denunciar la violencia implícita en cualquier proceso de racialización.

En el video "Bamba 45", Fabio Melecio Palacio recrea su historia personal para narrar una historia social; la de las duras condiciones de trabajo que viven los hombres negros que trabajan en las plantaciones azucareras. Este joven artista caleño utiliza los sonidos que producen los machetes de marca Bamba 45 de un grupo de corteros de caña de azúcar para llamar la atención sobre su precaria situación laboral. ${ }^{3}$ Estos sonidos que producen una música rítmica y estos utensilios que le resultan familiares, porque su padre era cortero, dan forma a su obra BMR (Bamba, Martillo y Refilón), una instalación de 582 machetes bruñidos por el uso, con la cual ganó el Premio Luis Caballero, uno de los más importantes del país en el campo de las artes plásticas. Del techo de la galería, convertido en un cañaveral invertido, cuelgan estas 582 hojas metálicas cuyo montaje se completa con un performance de afilado de machetes realizado por él y dos compañeros corteros, y un video de las plantaciones. Su obra pone en evidencia sin estetizaciones superfluas, la invisibilidad social de las dispendiosas y esforzadas jornadas de trabajo de los corteros de caña, y al mismo tiempo, la profunda cadencia estética que acompaña su actividad.

A mi modo de ver, la singularidad y la fuerza emotiva del trabajo de estos dos artistas hace posible comprender las erosiones que se pueden hacer al orden racista y sexista a partir de nuevos modos de auto-representación y ejercicios políticos alternativos. En efecto, la lógica de estas discriminaciones no se juega toda en los niveles macrosociales y por el contrario su fuerza reside en su capacidad de articular distintos niveles del poder que pueden minarse desde adentro, como lo hacen Angulo y Palacios, si

3 La denuncia de esta situación laboral dio lugar a una prolongada huelga en el segundo semestre de 2008. 
atendemos a la relativa autonomía que pueden desplegar para subvertir en los niveles microfísicos, la "colonialidad del poder".

\section{Referencias bibliográficas}

BHABHA, Homi. El lugar de la cultura. Buenos Aires, Ediciones Manantial SRL, 2002 [1994].

BOESTEN, Jelke. Narrativas de sexo, violencia y disponibilidad:Raza, género y jerarquías de la violación en Perú. En: WADE, Peter; URREA, Fernando y VIVEROS, Mara. Raza, etnicidad y sexualidades: ciudadanía y multiculturalismo en América Latina. Bogotá, Universidad Nacional de Colombia, 2008, pp.119-220.

BUTLER, Judith. Humain, Inhumain. Le travail critique des normes. Paris, éditions Amsterdam, 2005.

CASTRO-GómEZ, Santiago. Michel Foucault y la colonialidad del poder. Tabula Rasa. Bogotá, Colombia, n 6, enero-junio 2007, pp.153-172.

CÉSAIRE, Aimé. Discurso sobre el colonialismo. En: CÉSAIRE, Aimé. Discurso sobre el colonialismo. Madrid, Akal, 2006, pp.13-44.

DE LA CADENA, Marisol. Las mujeres son más indias. Espejos y Travesías. Antropología y Mujer en los 90. Ediciones Isis Internacional $\mathrm{n}^{\circ} 16$, Santiago, 1992, pp.25-45.

FouCAUlt, Michel. Sujeto y Poder. Bogotá, Carpe Diem,1991.

Foucault, Michel. Defender la Sociedad. Curso en el Collège de France (1975-1976). México, Fondo de Cultura Económica, 2001.

SAÏD, Edward. Orientalismo. Barcelona, Nuevas Ediciones de Bolsillo, 2003 [1978].

STOLER, Anne Laura. Genre et moralité dans la construction impériale de la race. Actuel Marx, 18, Paris, PUF, 2005, pp.75-103.

STOLER, Anne Laura. Race and the Education of Desire. Foucault's History of Sexuality and the Colonial Order of Things. Durham \& London, Duke Unicversity Press, 1995. 
reflexiones a partir de una experiencia investigativa en Colombia

TAMALE, Sylvia. Researching and theorising sexualities in Africa. En: TAmale, Sylvia. (ed.) African Sexualities. A reader. Cape Town, Dakar, Nairobi and Oxford, Pambazuka Press, 201, pp.11-36.

Viveros VigoyA, Mara y GIL HeRNÁNDEZ, Franklin. Género y generación en las experiencias de ascenso social de personas negras en Bogotá. Maguaré, n 24, 2010, pp.99-130.

VIVEROS VIGOYA, Mara. De quebradores y cumplidores: sobre hombres, masculinidades y relaciones de género en Colombia. Bogotá, CES, Universidad Nacional de Colombia, Fundación Ford, Profamilia Colombia, 2002a.

Viveros VigoyA, Mara. El gobierno de la sexualidad juvenil y la gestión de las diferencias. Reflexiones a partir de un estudio de caso colombiano. Revista Colombiana de Antropología, vol. 40, enerodiciembre de 2004, pp.155-183.

Viveros VigoyA, Mara. (coord.) Informe de la investigación HexcaColombia, Enero 19, 2011.

Viveros Vigoya, Mara. Sexuality and Desire in Racialized Contexts. In: AgGleton, Peter; BOYCE, Paul; MOORE, Henrietta L; PARKER, Richard. (ed.) Understanding Global Sexualities. New Frontiers. London, New York, Routledge, 2012, pp.218-231.

VIVEROS VigOYA, Mara. Movilidades y desigualdades espaciales y sociales en el contexto del multiculturalismo latinoamericano. Una lectura en clave de género. En: STRÖBELE-GREGOR, Juliana y WOLLRAD, Dörte. (ed.) Espacios de género. Buenos Aires, Nueva Sociedad/Fundación Friedrich Ebert/Adlaf, 2013, pp.189-203.

WADE, Peter. Gente negra, nación mestiza. Dinámicas de las identidades raciales en Colombia. Bogotá, Editorial Universidad de Antioquia/ ICAN / Siglo del Hombre Editores/ Ediciones Uniandes, 1997.

WADE, Peter. Race and Sexuality in Latin America. London, Pluto Press, 2009. 